\title{
New-Onset Refractory Status Epilepticus Mimicking Herpes Virus Encephalitis
}

\author{
Gianfranco Puoti $^{a} \quad$ Andrea Elefante $^{c}$ Dario Saracino ${ }^{a}$ \\ Antonella Capasso $^{b} \quad$ Roberto Cotrufo $^{a} \quad$ Clara Belluomo Anello $^{b}$ \\ ${ }^{a}$ I Division of Neurology, Department of Clinical and Experimental Medicine, and \\ ${ }^{b}$ Intensive Care Unit, Department of Emergency Anesthesiology and Surgery, \\ Second University of Naples, and ' Neuroradiology, Department of Diagnostic Imaging, \\ University 'Federico II', Naples, Italy
}

\section{Key Words}

New-onset refractory status epilepticus $\cdot$ Status epilepticus $\cdot$ Encephalitis

\begin{abstract}
New-onset refractory status epilepticus (NORSE) is a recently defined clinical entity that describes patients who present with status epilepticus of unclear etiology that is highly refractory to therapy. Magnetic resonance imaging (MRI) of NORSE usually discloses no specific abnormalities except for an occasional mild T2/FLAIR hyperintense signal of the mesial temporal lobe. Here, we report a peculiar case of NORSE in which brain MRI showed massive alteration of both temporal lobes, with features strongly supporting the diagnosis of herpes virus encephalitis, but lacking any laboratory evidence of viral infection in the blood and cerebrospinal fluid. It showed also striking signal alterations in the thalamus, which got worse in the course of the disease. This report emphasizes the possibility that seizure activity alone plays a critical role in both determining the disease and whether it will be selfsustaining.

(c) 2013 S. Karger AG, Basel
\end{abstract}

\section{Introduction}

New-onset refractory status epilepticus (NORSE) is a recently defined clinical entity that describes patients who present with status epilepticus (SE) of unclear etiology that is highly refractory to standard first- and second-line therapeutic agents [1]. 
Puoti et al.: New-Onset Refractory Status Epilepticus Mimicking Herpes Virus Encephalitis

A variety of terms have been used in the literature to refer to this syndrome, including 'de novo cryptogenic refractory multifocal febrile status epilepticus' [2], 'idiopathic catastrophic epileptic encephalopathy' [3], 'status epileptics owing to presumed encephalitis' [4], 'devastating epilepsy in school-age children' (DESC) [5], acute nonherpetic encephalitis with refractory repetitive partial seizures' [6], 'acute encephalitis with refractory repetitive partial seizures' (AERRPS) [7], and 'febrile infection-related epilepsy syndrome' (FIRES) [8, 9].

NORSE does not appear to be related to obvious risk factors for epilepsy such as contributing familial or personal anamnesis or previous brain trauma, and unspecific febrile illness is often reported in the weeks preceding SE. Common features of NORSE are female gender, young age (average 28-30 years), previous good health, unrevealing instrumental workup and poor outcome [1]. Among investigated cases, the mortality is close to $50 \%$.

A large number of hypotheses have been proposed to explain the pathogenesis of NORSE, but none have proven to be conclusive. Inflammation, by means of interleukins IL-1 $\beta$ and TNF- $\alpha$, has been pointed out as a possible trigger for neuronal hyper-excitability by altering neurotransmitter levels and receptor response. However, in most cases no clear causes of inflammation are present and neuropathology does not provide any exhaustive answers.

In the absence of a defined pathogenic mechanism for NORSE, when a patient presents with refractory SE, especially if preceded by or in association with a febrile illness, the hypothesis of infectious encephalitis cannot be rejected. NORSE occurs in 10-20\% of patients with etiologically proven infectious encephalitis, but in clinical practice the suspicion of encephalitis is also raised with suggestive cerebrospinal fluid (CSF) and magnetic resonance imaging (MRI) alterations (so-called bona fide encephalitis), which are often found in patients affected by NORSE. So, lacking confirmatory postmortem data, the link between NORSE and central nervous system infections is hard to demonstrate but cannot be excluded.

Here, we report a peculiar case of NORSE associated with altered brain MRI scans, showing features strongly supportive of the diagnosis of herpes virus encephalitis, but lacking any laboratory evidence of viral infection in the blood and CSF. In this report, after deep analysis of neurological images, we conclude that all the observed magnetic resonance signal alterations could represent the tissue response to the continuous epileptic firing, further emphasizing NORSE as a possible primary malignant epilepsy.

\section{Case Presentation}

A 41-year-old man presented with a parainfectious syndrome characterized by lowgrade fever and vomiting. Within 3 days, he experienced increasingly complex partial epileptic seizures, rapidly changing from tonic-clonic seizures to generalized SE, requiring intubation and admission to the intensive care unit. The patient had borderline intellectual functioning, possibly the expression of very mild perinatal posthypoxic encephalopathy. Clinical testing at the time of admission (as compared with neuroimaging and electroenchephalograpic studies which had been performed when the patient was a child) did not disclose any anatomic brain changes, pathology, or abnormal electric activity. No seizures were ever reported until the onset of SE.

The neurological examination, performed in the intensive care unit, revealed no cranial nerve or focal changes, or meningism. Because first-line anticonvulsants were not able to achieve the clinical remission of SE, intravenous anesthetic anticonvulsants were required. 
Puoti et al.: New-Onset Refractory Status Epilepticus Mimicking Herpes Virus Encephalitis

Intravenous infusions of propofol or thiopental were used to maintain the patient in an electrographic burst-suppression pattern, while enteral antiepileptic medications were titrated to therapeutic doses (e.g. valproic acid, clobazam, topiramate, phenobarbital, carbamazepine, and levetiracetam) [10, 11]. Extensive laboratory analyses, including virological, immunological, paraneoplastic, mitochondrial and endocrine tests were performed on blood and CSF with no evidence of infectious, inflammatory, mitochondrial, autoimmune, neoplastic and paraneoplastic pathology. No elevated proteins or pleocytosis were detected in CSF. Brain MRI, carried out 3 days after SE onset, showed signal hyperintensity of the temporal lobe bilaterally and symmetrically in T2 and FLAIR sequences, with involvement of both the cortex and white matter, deep into the hippocampus and pulvinar (fig. 1). Signs of laminar necrosis were detected in the superior and medium temporal giri bilaterally (fig. 1a, d, e). Magnetic resonance diffusion-weighted images, including apparent diffusion coefficient mapping, permitted us to identify the presence of vasogenic edema (fig. 1h, i). Although some brain MRI findings strongly suggested herpes simplex virus (HSV) encephalitis (i.e. topography of lesions and cortical involvement), the absence of MRI signs of parenchymal hemorrhagic necrosis along with the absence of pleocytosis and hyperproteinorrachia in CSF and viral HSV1 and HSV6 RNA in blood and CSF did not permit the confirmation of that diagnosis. Therefore, a presumptive diagnosis of encephalitis with SE was considered, and acyclovir was empirically added to antibiotics (ceftriaxone, vancomycin) and steroids.

During the following days, each time the tapering of the maximal dose of intravenous anesthetic anticonvulsants was tried, SE began again. Follow-up brain MRI studies, performed within 4 weeks, demonstrated an unvaried neuroradiological picture, but with a reduction of vasogenic edema in the temporal lobes (fig. 1f) and more evident involvement of the pulvinar bilaterally (fig. 1g).

The patient died 41 days after the onset of SE and an autopsy was not performed. Written informed consent could not be obtained from the deceased patient. Nonetheless, we acquired written informed consent from the patient's parents for the publication of this case report and the accompanying images.

\section{Discussion}

Our case seems to show remarkable clinical similarity to most NORSE cases reported in the literature, i.e. the presence of febrile illness with no preceding condition, negative laboratory studies including CSF analysis, SE refractory to conventional pharmacotherapy, and fatal prognosis.

Magnetic resonance features characterize the diversity of our case. As for the literature, initial MRI of NORSE usually discloses no specific abnormalities except for occasional T2/FLAIR hyperintense signal of the mesial temporal lobe, and often reveals generalized atrophy and bilateral hippocampus enhancement in follow-up studies [12]. Other authors have reported leptomeningeal enhancements and signal hyperintensities in the peri-insular region [9]. Our case is the first one to show a so evidently massive involvement of brain parenchyma by neuroimaging, i.e. bilateral involvement of the entire temporal lobe (cortex and white matter), extending from the temporal pole up to the hippocampus and pulvinar, with signs of vasogenic edema and laminar necrosis. The main question is: what is the etiopathogenic basis of such alterations? At first glance, just looking at the MRI results, the picture seems strongly to suggest herpes encephalitis. The problem is that this kind of working diagnosis is generally made on the basis of (i) CSF pleocytosis (usually lympho- 
Puoti et al.: New-Onset Refractory Status Epilepticus Mimicking Herpes Virus Encephalitis

cytes) as a specific response to the pathogenic agent; (ii) CSF protein increase and expression of blood-brain barrier failure, and (iii) CSF isolation of the specific virus by polymerase chain reaction. All 3 of these elements were absent in our case. Even though the virus and/or its nucleic acid are frequently not isolated, also not uncommonly for encephalitis without associated seizure activity, the absence of pleocytosis and hyperproteinorrachia enabled us to exclude an infectious process [13]. So, we believe in the possibility that all the MRI changes observed in this case could be ascribed to the seizure activity itself.

Another point to be underlined is the MRI involvement of the medial thalamus bilaterally. Considering the extensive reciprocal connections with most cortical regions, including temporal, parietal, and insular cortices, its involvement does not surprise, and it further emphasizes the role of seizure activity as a key player and the possible role of the thalamus in seizure propagation and self-sustenance. Furthermore, the sole case reported with this feature [14] showed a neuropathological picture characterized by the presence of conspicuous neuronophagia with no evidence of infectious processes.

Perhaps neuropathology would have been useful to confirm the possibility that seizure activity itself was the cause of the MRI picture observed in our case; nonetheless, to date no pathognomonic neuropathological features have been reported by any group in the literature, and, apart from the abovementioned report [14], only 1 report described gliosis in the absence of inflammation [8] as a possible expression of a reactive process, that is to say a process secondary to the seizure activity itself.

\section{Conclusion}

Though an underlying etiology remains unproven in the patient hereby reported, as in many other similar cases, and it cannot be known if and/or how much the possible mild perinatal post-anoxic encephalopathy influenced the onset and/or self-sustaining nature of SE in our patient, we state that NORSE is an etiologically heterogeneous nosological entity with a significant proportion of cases due to noninfectious causes, in which the seizure activity itself plays a role in both determining the disease and its self-sustaining nature. Further study of this poorly understood form of SE is needed, in particular, the exact definition of its biomolecular basis.

\section{Disclosure Statement}

None of the authors had any conflicts of interest including any financial (grant or funding), personal or other relationships with other people or organizations that could inappropriately influence or bias their work.

\section{References}

1 Costello DJ, Kilbride RD, Cole AJ: Cryptogenic new onset refractory status epilepticus (NORSE) in adults infectious or not? J Neurol Sci 2009;277:26-31.

2 Van Lierde I, Van Paesschen W, Dupont P, Maes A, Sciot R: De novo cryptogenic refractory multifocal febrile status epilepticus in the young adult: a review of six cases. Acta Neurol Belg 2003;103:88-94.

-3 Baxter P, Clarke A, Cross H, Harding B, Hicks E, Livingston J, Surtees R: Idiopathic catastrophic epileptic encephalopathy presenting with acute onset intractable status. Seizure 2003;12:379-387. 
Puoti et al.: New-Onset Refractory Status Epilepticus Mimicking Herpes Virus Encephalitis

4 Kramer U, Shorer Z, Ben-Zeev B, Lerman-Sagie T, Goldberg-Stern H, Lahat E: Severe refractory status epilepticus owing to presumed encephalitis. J Child Neurol 2005;20:184-187.

5 Mikaeloff Y, Jambaqué I, Hertz-Pannier L, Zamfirescu A, Adamsbaum C, Plouin P, Dulac O, Chiron C: Devastating epileptic encephalopathy in school-aged children (DESC): a pseudo encephalitis. Epilepsy Res 2006;69:67-79.

-6 Awaya Y, Fukuyama Y, Hayashi K, Osawa M: Acute non-herpetic encephalitis with severe refractory status epilepticus - its overwhelming ictogenicity, epileptogenicity, long-term prognosis and review of the literature. No To Hattatsu 2007;39:138-144.

7 Sakuma H, Awaya Y, Shiomi M, Yamanouchi H, Takahashi Y, Saito Y, Sugai K, Sasaki M: Acute encephalitis with refractory, repetitive partial seizures (AERRPS): a peculiar form of childhood encephalitis. Acta Neurol Scand 2010;121:251-256.

-8 van Baalen A, Häusler M, Boor R, Rohr A, Sperner J, Kurlemann G, Panzer A, Stephani U, Kluger G: Febrile infection-related epilepsy syndrome (FIRES): a nonencephalitic encephalopathy in childhood. Epilepsia 2010;51:1323-1328.

-9 Kramer U, Chi CS, Lin KL, Specchio N, Sahin M, Olson H, Nabbout R, Kluger G, Lin JJ, van Baalen A: Febrile infection-related epilepsy syndrome (FIRES): pathogenesis, treatment, and outcome: a multicenter study on 77 children. Epilepsia 2011;52:1956-1965.

10 Abend NS, Dlugos DJ: Treatment of refractory status epilepticus: literature review and a proposed protocol. Pediatr Neurol 2008;38:377-390.

11 Striano P, Elefante A, Coppola A, Tortora F, Zara F, Minetti C, Striano S: Dramatic response to levetiracetam in post-ischaemic Holmes' tremor. J Neurol Neurosurg Psychiatry 2007;78:438-439.

-12 Ismail FY and Kossoff EH: AERRPS, DESC, NORSE, FIRES: multi-labeling or distinct epileptic entities? Epilepsia 2011;52:185-189.

Barry E, Hauser WA: Pleocytosis after status epilepticus. Arch Neurol 1994;51:190-193.

Boyd JG, Taylor S, Rossiter JP, Islam 0, Spiller A, Brunet DG: New-onset refractory status epilepticus with restricted DWI and neuronophagia in the pulvinar. Neurology 2010;74:1003. 


\section{Case Reports in Neurology}
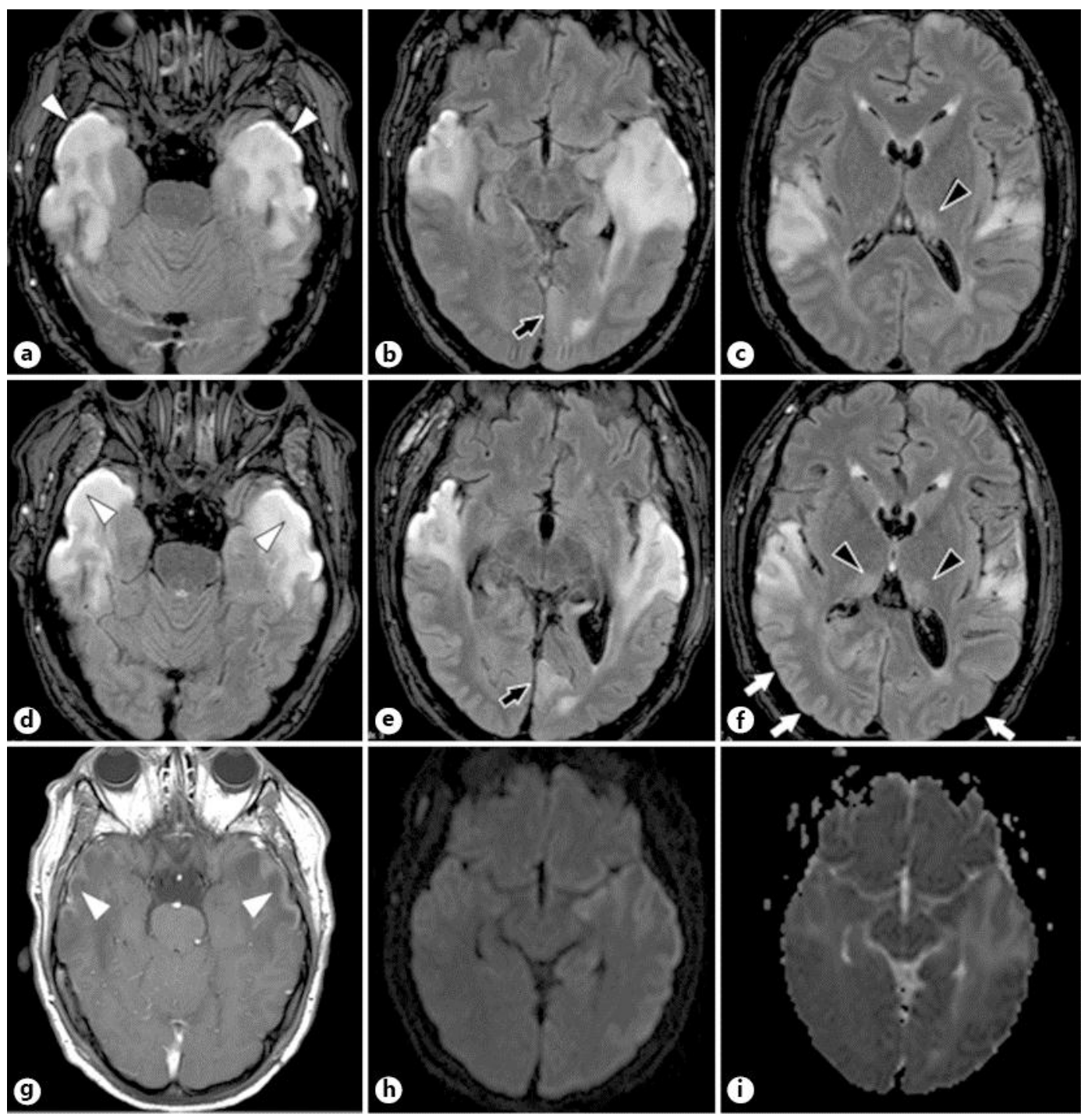

Fig. 1. MRI features. FLAIR images $(\mathbf{a}-\mathbf{c})$, contrast-enhanced T1-weighted image (g), diffusion-weighted image (h), and apparent diffusion coefficient map (i) performed 3 days after SE onset. $\mathbf{d}$-f FLAIR images 1 month later. Evidence of bilateral involvement of the entire temporal lobe (cortex and white matter), from the temporal pole up to the hippocampus, with signs of laminar necrosis ( $\mathbf{a}, \mathbf{d}$, white arrowheads), is easily recognizable after gadolinium (g). The edema is significantly more prominent 3 days after SE onset (a, b), compared with 1 month later (e). The high signal in the apparent diffusion coefficient map demonstrates it is vasogenic ( $\mathbf{h}, \mathbf{i}) . \mathbf{b}, \mathbf{e}, \mathbf{f}$ Cortical involvement outside the temporal lobe (f, white arrows), in particular of the left precuneus (black arrows), is far more evident in the second MRI. c, $f$ The left pulvinar shows hyperintensity, which increased 1 month later, with involvement of the contralateral nucleus (black arrowheads). 